\title{
Impacto Económico de la Medicina Familiar en los Sistemas de Salud de Iberoamérica
}

\section{Economic Impact of Family Medicine on Health Systems in Ibero-America}

\section{Impacto Econômico da Medicina de Família nos Sistemas de Saúde na América Latina}

\section{Grupo de trabajo:}

Urquiza Raúl (Argentina); Nermen Nulvio (Brasil); Aguayo Arteaga Fátima (Colombia); Munive Mery (Costa Rica); Gerardo Ramírez (México); Rivera Patricia (Panamá); Chunga Juan Carlos(Perú); Ortiz Sonia (Puerto Rico); Barrios Anahi (Uruguay); Laura Jaramillo (Colombia); Cipolla Giulia (Paraguay).

\section{Resumen}

Son escasos los estudios que abordan la importancia, económica y sanitaria, que tiene el modelo organizativo de un nivel asistencial o la presencia de determinados profesionales. El objetivo del presente estudio, de carácter descriptivo y transversal, fue explorar y analizar las posibles asociaciones entre la especialidad de medicina familiar e indicadores económicos y sanitarios en 16 países de Iberoamérica. El procesamiento de datos fue realizado a través del programa $R$, un lenguaje de programación que muestra "un conjunto de funciones que mantiene algún tipo de relación entre ellas". Se observa una asociación en positivo, del número de especialistas de medicina familiar con el PIB, la inversión en salud y la esperanza de vida y en negativo con el índice GINI, la anemia, la mortalidad en menores de 5 años, la razón de mortalidad materna y la mortalidad en accidentes en tránsito. EI PIB per cápita se relaciona negativamente con la anemia, la mortalidad en menores de 5 años, razón de mortalidad materna y por accidentes y menos intensamente con la mortalidad cardiovascular y el suicidio. No se observan correlaciones con el gasto de bolsillo o la inversión en sanidad. A pesar de las diferentes realidades socio sanitarias de los países estudiados se objetiva una relación favorable entre la disponibilidad de especialistas en Medicina Familiar y mejores resultados en salud lo que sugiere que puede ser una estrategia eficiente para los servicios sanitarios. Son necesarios más estudios que analicen el alcance estadístico de esta asociación.

Palabras clave: Medicina Familiar; Atención Primaria; Eficiencia; Economía de la Salud

Cómo citar: Edgar L, Salvador T, José M. Impacto Económico de la Medicina Familiar en los Sistemas de Salud de Iberoamérica. Rev Bras Med Fam Comunidade. 2018;13(Suppl 1):43-53. http://dx.doi.org/10.5712/rbmfc13(40)1852

\author{
León Edgara \\ Tranche Salvador ${ }^{b}$ \\ Montano Joséc
}

\author{
a Universidad Católica del Ecuador; \\ Confederación Iberoamericana de Medicina \\ Familiar (CIMF). Ecuador. \\ edgarleonseg@gmail.com \\ (Autor correspondiente) \\ b Universidad de Oviedo (UNIOVI), Facultad \\ de Medicina de Oviedo. España. \\ stranchei@gmail.com \\ c Universidad del Valle Colombia (UNIVALLE). \\ Colombia. jose.ivo.mc@gmail.com
}




\begin{abstract}
There are few researches that address the economic and sanitary importance of the organizational model of a level of care or of the presence of certain professionals. The aim of this descriptive and transversal study is to explore and analyze the possible associations between the specialty of family medicine and economic and sanitary indicators in 16 countries of Ibero-America. The data processing was carried out through the program $R$, a programming language that shows "a set of functions that maintain some type of relation between them". It seems that there is a positive association between the number of specialists in family medicine with GDP, investment in health and life expectancy and in negative with the GINI index, anemia, mortality in children under 5 years, maternal mortality ratio and in traffic accidents. The GDP per capita is negatively related to anemia, mortality in children under 5 years of age, maternal and accident mortality ratio, and less intensely with cardiovascular mortality and suicide. There are no correlations between pocket expenses or investment in healthcare. Despite the different health and social realities of the countries studied, a favorable relation is found between the availability of specialists in Family Medicine and better health results, which suggests that it can be an efficient strategy for health services. More studies are necessary to analyze the statistical scope of this association.
\end{abstract}

Keywords: Family Medicine; Primary Health Care; Efficiency; Health Economics

\title{
Resumo
}

São poucos os estudos que abordam a importância, economica e a sanitária, que tem o modelo organizacional de um nível de atenção ou a presença de determinados profissionais. $O$ objetivo do presente estudo, de caráter descritivo e transversal, foi explorar e analisar as possíveis associações entre a especialidade de medicina de família e indicadores econômicos e de saúde em 16 países da lbero-América. O processamento de dados foi realizado através do programa $R$, uma linguagem de programação que mostra "um conjunto de funções que mantém algum tipo de relação entre elas". Existe uma associação positive em relação ao número de especialistas em medicina de família com o PIB, investimento em saúde e expectativa de vida e em negativo com o índice GINI, anemia, mortalidade em crianças menores de 5 anos, a razão de mortalidade materna e mortalidade em acidentes em trânsito. O PIB per capita está negativamente relacionado à anemia, mortalidade em crianças menores de 5 anos, taxa de mortalidade materna e por acidentes e menos intensamente com mortalidade cardiovascular e o suicídio. Não se observaram correlações com despesas reembolsáveis ou investimentos em assistência médica. Apesar das diferentes realidades sociais e de saúde dos países estudados, uma relação favorável é encontrada entre a disponibilidade de especialistas em Medicina de Família e melhores resultados em saúde, o que sugere ser esta uma estratégia eficiente para os serviços sanitários. Mais estudos são necessários para analisar o escopo estatístico desta associação.

Palavras-chave: Medicina de Família; Atenção Primária; Eficiência; Economia da Saúde

\section{Introducción}

La Organización Mundial de la Salud (OMS) en su Informe de la Salud en el mundo 2003, afirma que un sistema de salud asentado en la atención primaria deberá incorporar los principios de Alma Ata de "Equidad, acceso universal, participación de la comunidad y acción intersectorial". Deberá tomar en consideración cuestiones sanitarias generales de ámbito poblacional y organizará una asistencia integrada que vinculará la prevención, la atención a enfermos agudos y la atención a enfermos crónicos en todos los elementos del sistema sanitario. Evaluará continuamente la situación para procurar mejorar el desempeño". Sin embargo, hoy 40 años después, la atención primaria no ha alcanzado en ningún país ni el desarrollo suficiente ni, por supuesto, los objetivos planteados.

Los retos a los que se enfrentan los sistemas sanitarios en todo el mundo y específicamente en el ámbito iberoamericano, son formidables. El desafío más importante pasa por adecuar los servicios de salud a las necesidades de los ciudadanos, lo que obliga a reducir las desigualdades en los resultados en salud y, al tiempo que se asegura la sostenibilidad, procurar también incrementar la financiación de los sistemas sanitarios. ${ }^{2}$

Estos desafíos obligan a profundizar en el análisis económico riguroso incorporando al mundo sanitario instrumentos e indicadores económicos que comparen el producto de los servicios de salud 
con los costes. De ello resultan análisis como coste-efectividad, coste-beneficio y coste-utilidad de las intervenciones, que facilitan una mejor asignación de recursos y por tanto sirven de base para contabilizar y evaluar el coste de las prestaciones, planificar la toma de decisiones y analizar el financiamiento de los sistemas de Salud. Existen varios mecanismos y varios tipos de análisis económicos útiles para la medición de impactos económicos, como costeo directo, predeterminado, por absorción, real normalizado, real integrado y $A B C$ entre otros. Cada uno de estos, sin embargo, puede presentar grandes diferencias para una misma enfermedad o prestación; incluso si se analizan grupos etarios comunes, las variaciones pueden ser muy amplias. ${ }^{3}$

La mayoría de los estudios relacionados con evaluaciones económicas se han realizado en procesos concretos o actividades puntuales, por lo que analizar la implicación económica de un nivel de atención en un determinado sistema de salud es un proceso complejo y con variables indirectas. Se realizó un estudio exploratorio sobre la asociación que la Medicina Familiar tiene en los sistemas de salud de los países que conforman la Confederación Iberoamericana de Medicina Familiar.

En estudios previos, también otros autores como B. Starfield y J. Macinko ${ }^{4,5}$ en Estados Unidos (EUA) demostraron que la dotación de médicos de atención primaria se asoció con mejores resultados de salud, en mortalidad por todas las causas, cáncer, enfermedad cardíaca, accidente cerebrovascular y mortalidad infantil. También demostraron que en Europa, el PIB y número de médicos de familia, se asoció de forma independiente, con reducciones en los años de vida potenciales perdidos y con la mortalidad por todas las causas. ${ }^{6}$

\section{Métodos}

Se trata de un estudio descriptivo y transversal que explora la relación entre indicadores sanitarios y económicos con la presencia de especialistas de medicina familiar en los sistemas sanitarios de 16 países de Iberoamérica.

En base a la disponibilidad de indicadores y a informes de la OMS,$^{1}$ de los Ministerios de Salud de los diferentes países, evaluaciones de Sociedades Científicas, Eurostat ${ }^{7}$ y el Banco Mundial ${ }^{8}$ se eligieron los siguientes:

\section{Indicadores Macroeconómicos}

Producto Interior Bruto(PIB). Es el indicador más relevante para medir la actividad económica y la evolución económica de un país y además sirve de referente para comparar la situación económica del país con la región. Se incluye también el porcentaje del PIB destinado a Sanidad en cada país.

Índice de GINI. Es el indicador más utilizado a nivel mundial para medir la desigualdad de los ingresos (y para cualquier otro tipo de desigualdad). Puede oscilar entre el 0 que expresa la igualdad perfecta y el valor de 100 para la máxima desigualdad posible. ${ }^{9}$

Gasto de bolsillo. Se considera como tal al desembolso realizado por las familias en el último año para atención médica, medicamentos, pruebas complementarias, etc. 


\section{Indicadores Sanitarios}

- Número de Médicos de Familia por 100.000 habitantes.

- Expectativa de vida. o esperanza de vida.

- Enfermedades Trazadoras. Para la Organización Panamericana de la Salud (OPS) existen enfermedades trazadoras que pueden ser representativas del nivel sanitario de un país. Son las siguientes: prevalencia de anemia en niños en menores de 5 años, razón de mortalidad materna (número de defunciones maternas por 100.00 nacidos vivos); tasa de mortalidad en menores de 5 años, mortalidad por enfermedades cardiovasculares y mortalidad por suicidio y por accidentes de tránsito.

Se exploró la relación entre los indicadores económicos, los indicadores de salud y el indicador de disponibilidad de MF para 16 países miembros de CIMF: Argentina, Bolivia, Brasil, Chile, Colombia, Costa Rica, República Dominicana, Ecuador, España, México, Nicaragua, Panamá, Perú, Portugal, Paraguay y Uruguay ${ }^{6}$ Los datos corresponden al año 2015 siendo este el último año con datos completos para todos los países. El procesamiento estadístico se realizó con el lenguaje de programación y análisis estadístico $\mathrm{R}$ ( $\mathrm{R}$ Core Team, 2017). ${ }^{10} \mathrm{R}$ es un lenguaje de programación que viene equipado con un conjunto de herramientas destinadas al cálculo y generación de gráficos estadísticos que evidencia "un conjunto de funciones que mantienen algún tipo de relación entre ellas".

Al tratarse de una revisión sin participación de pacientes no se solicitó aprobación a ningún Comité Ético de Investigación. En cualquier caso los procedimientos se han ajustado a las normas éticas de la Asociación Médica Mundial y la Declaración de Helsinki.

\section{Resultados}

La Tabla 1, recoge 12 indicadores que nos pueden permitir comparar la situación sanitaria y económica entre los diferentes países.

Antes de proceder al análisis de la figura 1, resulta pertinente aclarar que el tamaño del círculo, el color y la intensidad del mismo, marcan la relación entre variables. $Y$ así el color azul indica una relación directa y el color rojo una relación inversa. A mayor tamaño del circulo es mayor la asociación, al igual que la intensidad del color (a más intenso mayor relación). Se puede ver cómo el tamaño del círculo, la intensidad del color y en éste caso el color azul, son máximos cuando se comparan entre sí las mismas variables (MF con MF; PIB con PIB, etc.). Considerando el carácter exploratorio de este estudio y las dificultades metodológicas involucradas con este tipo de investigación, evaluase que el principal hecho no está en el grado de la asociación de per se, pero en la relación encontrada que tiene validez de contenido, considerando estudios precedentes. . $^{4}$

En la Figura 1 destaca la estrecha relación del número de médicos de familia en positivo con el PIB per cápita y la esperanza de vida y en negativo con el índice GINI, la anemia, la mortalidad en menores de 5 años, la razón de mortalidad materna y la mortalidad en tránsito. EI PIB per cápita se relaciona negativamente también con la anemia, la mortalidad en menores de 5 años, razón de mortalidad materna y por accidentes en tránsito y menos intensamente con la mortalidad cardiovascular y el suicidio. No se observan correlaciones o estas son muy, muy leves en otros indicadores como el gasto de bolsillo o la inversión en sanidad. 
Tabla 1. Indicadores Socio sanitarios por países.

\begin{tabular}{|c|c|c|c|c|c|c|c|c|c|c|c|c|}
\hline País & MF & PIB(pp) & GBolsillo & GINI & InvSalud & ExpVida & Anemia & MorMen & MorCVD & MorTransito & RMM & Suicidio \\
\hline ARG & 14,3 & $13.467,1$ & 30,7 & 42,7 & 4,8 & 78,3 & 21,4 & 11,6 & 17 & 14,1 & 52 & 14,2 \\
\hline BOL & 5 & 3.077 & 23,1 & 45,8 & 6,3 & 68,7 & 47,5 & 38,2 & 16 & 23,3 & 206 & 18,7 \\
\hline BRA & 2,7 & $8.757,2$ & 25,5 & 51,3 & 8,3 & 75,2 & 24,3 & 15,7 & 17 & 22,6 & 44 & 6,3 \\
\hline $\mathrm{CHL}$ & 5,6 & $13.653,2$ & 31,5 & 47,7 & 7,8 & 79,2 & 19,5 & 8,4 & 11 & 11,6 & 22 & 9,9 \\
\hline $\mathrm{COL}$ & 1,2 & $6.044,5$ & 15,4 & 51,1 & 7,2 & 74,2 & 26,8 & 15,8 & 15 & 18,9 & 64 & 6,1 \\
\hline CRI & 3,4 & $11.406,4$ & 24,9 & 48,2 & 9,3 & 79,6 & 28,5 & 9,1 & 11 & 14,9 & 25 & 0,7 \\
\hline RDOM & 0,1 & $6.468,5$ & 21,1 & 44,9 & 4,4 & 73,7 & 27,7 & 31,5 & 19 & 27,8 & 92 & 6,8 \\
\hline ECU & 6,2 & $6.150,2$ & 48,4 & 46,5 & 9,2 & 76,1 & 27,9 & 21,5 & 13 & 20,7 & 64 & 7,5 \\
\hline ESP & 74,8 & $25.787,9$ & 24 & 36 & 9 & 83,4 & 12,4 & 3,4 & 10 & 3,6 & 5 & 8,5 \\
\hline MEX & 34,2 & $9.152,9$ & 44 & 48,2 & 6,3 & 76,9 & 27,7 & 15 & 15 & 11,8 & 38 & 5 \\
\hline NIC & 1,7 & 2.096 & 37,5 & 46,6 & 9 & 75 & 28,4 & 20,3 & 16 & 14,9 & 150 & 9,5 \\
\hline PAN & 2 & 13.134 & 22,3 & 51 & 8 & 77,8 & 28,6 & 6,9 & 14 & 10,7 & 94 & 5,5 \\
\hline PER & 3,1 & $6.030,3$ & 28,6 & 44,3 & 5,5 & 74,7 & 32,8 & 16 & 13 & 13,3 & 68 & 5,8 \\
\hline PRT & 53,6 & $19.252,6$ & 26,8 & 35,6 & 9,5 & 81,5 & 12,8 & 3,5 & 11 & 7,7 & 0 & 13,6 \\
\hline PRY & 4,1 & $4.109,4$ & 49,4 & 48 & 9,8 & 73 & 25 & 20,6 & 18 & 23,4 & 132 & 10,2 \\
\hline URY & 14,6 & $15.524,8$ & 15,6 & 41,7 & 8,6 & 77,1 & 22,4 & 9,3 & 17 & 17,4 & 15 & 17 \\
\hline
\end{tabular}

Elaboración propia a partir de datos publicados por basados en datos obtenidos en OMS ${ }^{1}$, Eurostat ${ }^{7}$ y el Banco Mundial. ${ }^{8}$

ARG: Argentina; BOL: Bolivia; BRA: Brasil; CHL: Chile; COL: Colombia; CRI: Costa Rica; RDOM: República Dominicana; ECU: Ecuador; ESP: España; MEX: México; NIC: Nicaragua; PAN: Panamá; PER: Perú; PRT: Portugal; PRY: Paraguay y URY: Uruguay.

MF: número de especialistas en medicina familiar y comunitaria/100.000 habitantes; PIB(pp): producto interior bruto per cápita (en dólares); GBolsillo: Gasto de Bolsillo (en dólares); GINI: índice GINI; InvSalud: porcentaje PIB destinado a gasto sanitario; MorIMen: Mortalidad Menores de 5 años; ExpVida: expectativa de vida; MorCVD: mortalidad cardiovascular; MorTransito: Mortalidad por accidentes de Tránsito; RMM: Razón de Mortalidad Materna.

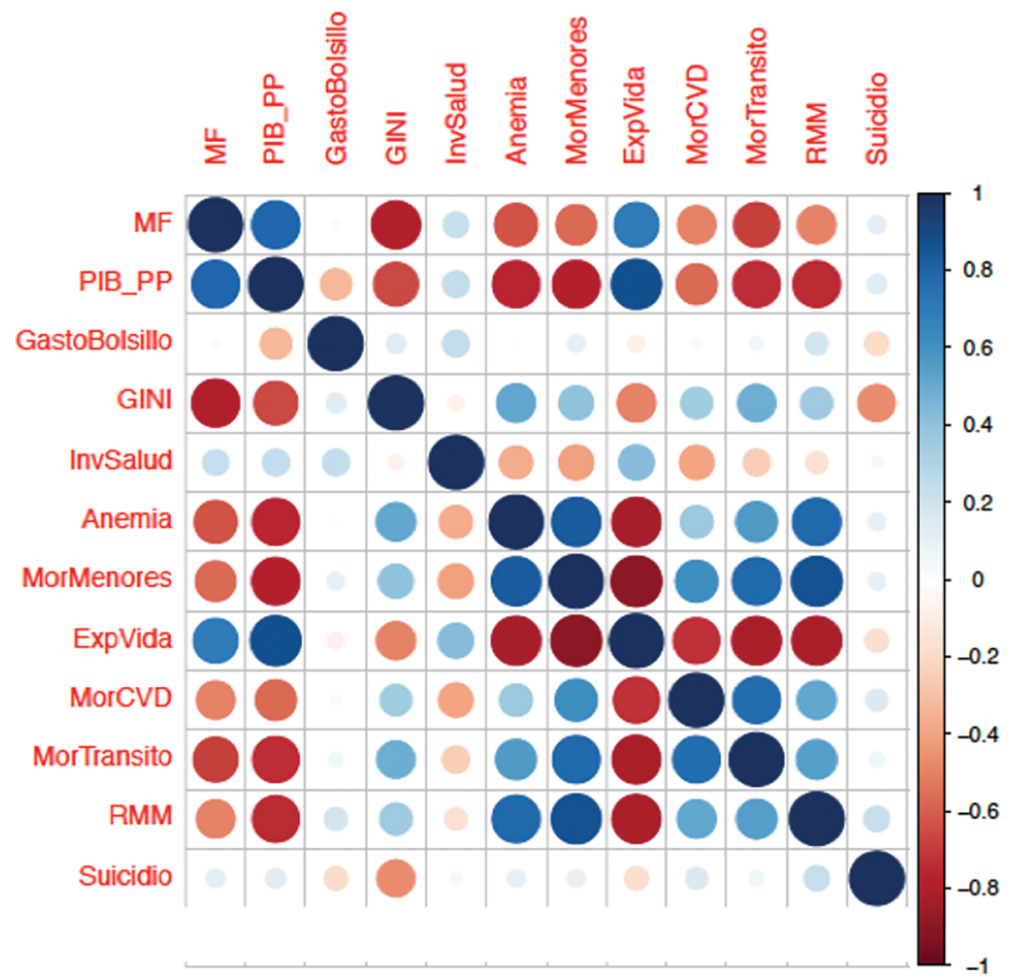

Figura 1. Relación entre los indicadores macroeconómicos y sanitarios.

MF: número de especialistas en medicina familiar y comunitaria/100.000 habitantes; PIB(pp): producto interior bruto per cápita (en dólares); GBolsillo: Gasto de Bolsillo (en dólares); GINI: índice GINI; InvSalud: porcentaje PIB destinado a gasto sanitario; Morımen: Mortalidad Menores de 5 años; ExpVida: expectativa de vida; MorCVD: mortalidad cardiovascular; MorTransito: Mortalidad por accidentes de Tránsito; RMM: Razón de Mortalidad Materna. 
La figura 2 corresponde a la matriz de gráficos de dispersión de la disponibilidad de Médicos Familiares y los indicadores económicos, permitiendo explorar la asociación entre estos, las escalas de cada uno de los indicadores están indicadas por los números ubicados en los bordes de la matriz. Los cuadros de la primera columna muestran como varían los indicadores económicos en función de la disponibilidad de Médicos Familiares. De manera recíproca los cuadros de la primera fila muestran como varía la disponibilidad de Médicos Familiares en función de los indicadores económicos. Los cuadros de la diagonal muestran histogramas de distribución de los datos de cada uno de los indicadores, el primer cuadro de arriba de la diagonal muestra que la mayoría de los países tienen cifras de disponibilidad de Médicos Familiares inferiores a 15 por 100.000 habitantes; el segundo cuadro de la diagonal muestra para el PIB per cápita una moda al rededor de US\$7.000; el tercer cuadro de la diagonal muestra para el gasto de bolsillo una moda alrededor de 20\%; el cuarto cuadro de la diagonal muestra que el índice de GINI tiene una moda alrededor de 45; por último el histograma del porcentaje del PIB invertido en salud la moda está entre 8 y 10.
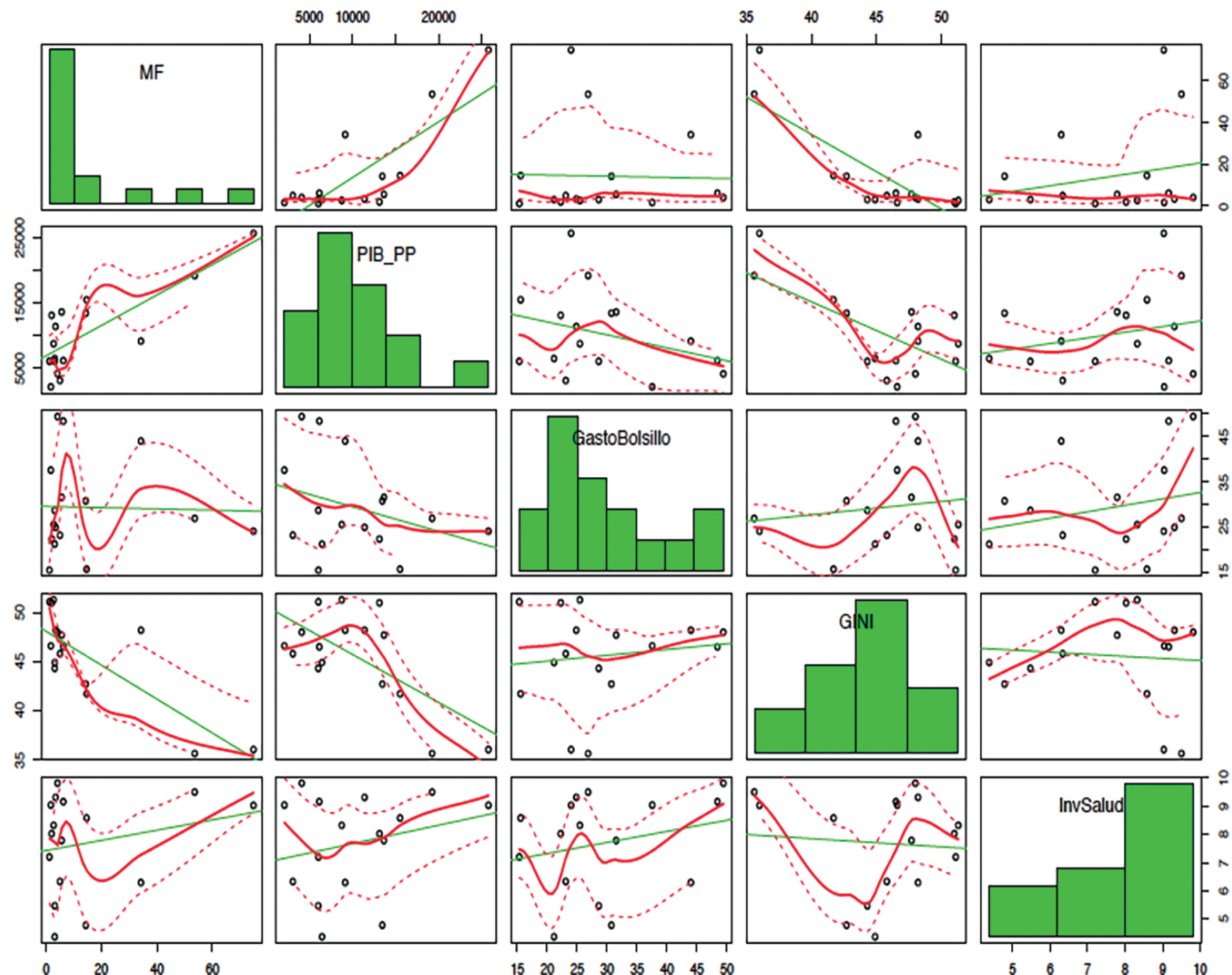

$\begin{array}{lllllllll}15 & 20 & 25 & 30 & 35 & 40 & 45 & 50\end{array}$

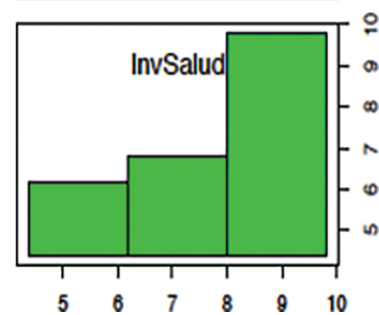

Figura 2. Matriz de gráficos de dispersión de MF con indicadores económicos.

MF: número de especialistas en medicina familiar y comunitaria/100.000 habitantes; PIB(pp): producto interior bruto per cápita (en dólares); GBolsillo: Gasto de Bolsillo (en dólares); GINI: índice GINI; InvSalud: porcentaje PIB destinado a gasto sanitario. 
La figura 3 permite explorar la asociación entre la cantidad o disponibilidad de médicos especialistas en Medicina Familiar y los principales indicadores socio sanitarios. Los cuadros de la primera columna muestran en su orden como varían los indicadores socio sanitarios en función de los Médicos Familiares, los cuadros de la diagonal muestra la distribución de los datos de cada uno de los indicadores.

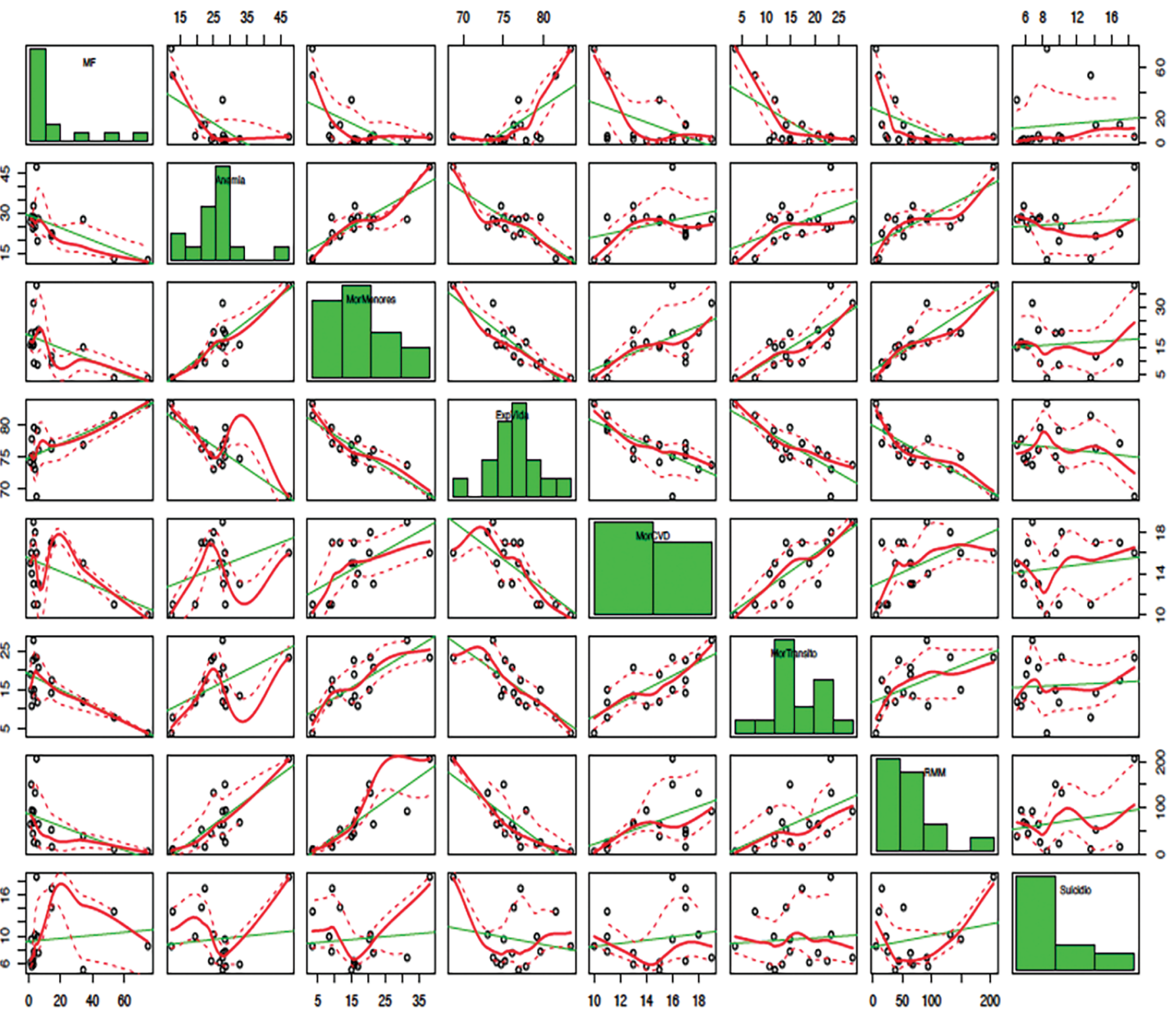

Figura 3. Matriz de gráficos de dispersión de MF con indicadores de salud.

MF: número de especialistas en medicina familiar y comunitaria/100.000 habitantes; PIB(pp): producto interior bruto per cápita (en dólares); GBolsillo: Gasto de Bolsillo (en dólares); GINI: índice GINI; InvSalud: porcentaje PIB destinado a gasto sanitario; MorIMen: Mortalidad Menores de 5 años; ExpVida: expectativa de vida; MorCVD: mortalidad cardiovascular; MorTransito: Mortalidad por accidentes de Tránsito; RMM: Razón de Mortalidad Materna.

\section{Discusión}

El presente trabajo es el primer análisis que explora la relación entre la disponibilidad de especialistas en Medicina Familiar, indicadores económicos y sanitarios entre los países que conforman la Confederación Iberoamericana de Medicina Familiar (CIMF). Se sugiere una asociación positiva entre la disponibilidad 
de médicos familiares y el PIB per cápita, lo que, a su vez, se traduce en mejoras notables en indicadores "duros" (mortalidad cardiovascular, mortalidad de menores de cinco años, esperanza de vida, entre otros) de salud pública.

Si el análisis se limitase sólo a la relación entre PIB per cápita y número de médicos de familia, podría equivocadamente malinterpretarse que la disponibilidad de Médicos Familiares es un "bien de lujo", es decir, sólo los países con mayor PIB y por tanto más ricos, pueden permitirse incrementar el número de médicos de familia. Sin embargo, esta relación no es lineal ya que a igualdad de PIB parece que una mayor disponibilidad de especialistas en Medicina Familiar, se asocia con un aumento de la expectativa de vida y con una reducción de la tasa de anemia y de la mortalidad en menores de cinco años; de la mortalidad cardiovascular; de la mortalidad por accidentes de tránsito y de la mortalidad materna.

Como mencionado anteriormente, los resultados encontrados coinciden con los resultados obtenidos por B. Starfield y J. Macinko. ${ }^{4-6}$ en Estados Unidos (EUA). Demostraron que la dotación de médicos de atención primaria se asoció con mejores resultados de salud, en mortalidad por todas las causas, cáncer, enfermedad cardíaca, accidente cerebro vascular y mortalidad infantil. Esta relación se mantuvo con independencia del año (1980-1995) o del nivel de análisis (estado, condado, área estadística metropolitana). Los resultados combinados para la mortalidad por todas las causas sugieren que un aumento de un médico de atención primaria por cada 10.000 habitantes, se asoció con una reducción promedio de la mortalidad de 5.3 por ciento. Los mismos autores demostraron que también en Europa, el PIB y número de médicos de familia, se asoció de forma independiente, con reducciones en los años de vida potenciales perdidos y con la mortalidad por todas las causas.

Más recientemente, Chetty y cols., ${ }^{11}$ también en EUA, describen que añadir un médico de familia más por 1.000 habitantes (o 100 por 100.000) ajustados los factores sociodemográficos, características de hospital y tasas de mortalidad, reduce los ingresos por neumonía, infarto agudo de miocardio e insuficiencia cardiaca un 7,5 y $8 \%$ respectivamente.

En cuanto a Europa, L. Vallejo ${ }^{12}$ éste mismo año publica una investigación basada en el seguimiento de una cohorte de personas mayores de 50 años residentes en Inglaterra, que fueron entrevistados cada dos años. Los datos corresponden a los periodos 2004-2005; 2006-2007 y 2008-2009. A los indicadores socio demográficos añadieron 35 indicadores de calidad de procesos que correspondían a 13 condiciones médicas. Estos indicadores se eligieron en base a la prevalencia, posibilidad de prevención y/o tratamiento, importancia en ancianos, posibilidad de su medición y potencial de mejora de su calidad. Son indicadores clínicos muy comunes en atención primaria (hipertensión, diabetes, dislipemia,) a los que añadieron dos indicadores de recursos: densidad de médicos de familia: número de médicos de familia por 1.000 habitantes y distancia hasta el centro de salud. Concluyeron que una mayor densidad de médicos de familia se asociaba con la calidad de cuidados y la distancia hasta el médico de familia presenta una asociación negativa (a mayor distancia perores resultados). Estos efectos se concentraron en enfermedades cardiovasculares, osteoartritis, diabetes, incontinencia y problemas auditivos. ${ }^{12}$

En nuestro trabajo destaca una relación negativa muy intensa entre el GINI y la disponibilidad de médicos de familia. La relación del PIB y la salud, así como el índice de GINI u otros índices de desigualdades de ingresos, se ha analizado en muchas publicaciones, pero sin relacionarlos con el modelo de organización sanitaria de cada país o la dotación de determinados profesionales. Jutz ${ }^{13}$ compara la 
situación en 42 países europeos y concluye que las desigualdades en los ingresos tienen más impacto en las desigualdades en salud que las políticas sociales. Bergquvist y cols., ${ }^{14}$ encontraron que el gasto social y de salud se asocian con mejores niveles de salud y menores desigualdades, aunque llaman la atención sobre la importancia de las políticas sanitarias (al igual que otros autores) ${ }^{15,16}$ Un reciente trabajo realizado en por Christopher, EUA, evalúa el efecto del gasto de bolsillo en sobre la desigualdad de ingresos. Describe que, en 2014, el índice de GINI era 47.84 y subió a 49.21 después de deducir los desembolsos médicos. Este gasto de bolsillo redujo el ingreso medio del decil más pobre en un 47,6\%, frente al 2,7\% del decil más rico, empujando a 7,013 millones de personas a la pobreza. ${ }^{17}$ En Brasil, Boing ${ }^{18}$ analiza los gastos de bolsillo según la encuesta de gastos familiares de 2002-2003 y 2008-2009, utilizando el criterio de pobreza del Banco Mundial (ingreso per cápita por día por debajo de US \$2,34 en 2002-2003 y de US \$3,54 en 2008-2009). El aumento de pobreza en los años 2002-2003 fue de 2,6 puntos porcentuales $(6,8 \%)$ y 2,3 puntos porcentuales $(11,6 \%)$ para los años 2008-2009. Este incremento se produjo a expensas del gasto en medicamentos. En nuestro caso no se ha encontrado asociación entre número de médicos de familia y gasto de bolsillo. No corresponde en este trabajo profundizar en los determinantes de salud y la relación entre factores económicos, sociales y la salud, máxime cuando esta relación es extraordinariamente compleja y no depende de un solo factor. ${ }^{19,20}$

El presente estudio presenta algunas limitaciones a ser llevadas en consideración:

1. La variabilidad existente entre los distintos países, tanto en indicadores económicos como sanitarios, dificulta la comparabilidad;

2. Los indicadores incluidos eran los disponibles para los 16 países y no ha sido posible incorporar otros determinantes sociales que quizá puedan explicar mejor las diferencias en los resultados en salud entre los países;

3. Es muy difícil medir el impacto de la medicina de familia en el sistema sanitario cuando muchos países de la región de lberoamérica (CIMF), disponen de menos de 10 médicos de familia por 100.000 habitantes;

4. El objetivo del presente trabajo se limita a explorar en conjunto los indicadores de médicos familiares, económicos y sanitarios, para lo cual se han usado herramientas gráficas, identificando algunas posibles asociaciones, sin embargo considerando que se trata de indicadores a nivel nacional se requiere aplicar otras herramientas estadísticas que permitan cuantificar las asociaciones observadas en futuros estudios.

En la VII Cumbre de CIMF celebrada recientemente en Cali se concluía que aunque la disponibilidad de médicos era un indicador grosero puesto que no aporta información sobre desempeño, funciones, ni roles, los resultados ponían de manifiesto que los mejores resultados en salud se obtenían en los países con mayor disponibilidad de especialistas en Medicina Familiar y mayor PIB per cápita por lo que recomendaban alcanzar la cifra mínima a corto plazo (9-10 años) de todos los países de la Confederación de 15 MF por 100.000 habitantes.

Para próximos estudios se sugiere incorporar otros indicadores que ayuden a analizar con mayor exactitud el papel de la medicina familiar en el sistema sanitario: cartera de servicios, capacidad de resolución, gasto sanitario en atención primaria versus otros niveles asistenciales, modelos organizativos de cada país. 


\section{Conclusiones}

Las realidades socio sanitarias de los países de la región son diversas y con improntas políticas que impactan directa e indirectamente en la salud de las poblaciones. Mientras las relaciones entre indicadores económicos como el gasto de bolsillo y el porcentaje de PIB de inversión en salud con los indicadores de salud muestran tendencias poco claras, destaca la relación favorable entre la disponibilidad de especialistas en Medicina Familiar y los resultados en salud sugiriendo que esta es una estrategia concreta, eficiente y al alcance de todos los países para convertir la inversión económica en resultados en salud.

La eficiencia clínica pasa por la maximización de la calidad de la atención y la satisfacción de los usuarios con los menores costes sociales posibles. El camino a la eficiencia social pasa por la efectividad clínica y eso parece estar íntimamente relacionado con el número y las características profesionales de la medicina de familia.

Debería ser motivo de reflexión en los círculos donde se debaten, establecen y evalúan las políticas y recursos del sector salud que la disponibilidad de especialistas en Medicina Familiar es un marcador consistente y sensible de calidad, equidad y eficiencia de los sistemas de salud.

Son necesarios más estudios, adecuadamente diseñados y con indicadores más precisos y homogéneos que permitan profundizar en el análisis de en lo que pueden aportar los especialistas de medicina familiar a la eficiencia de los servicios sanitarios y a la mejora de la salud de los ciudadanos.

\section{Referencias}

1. Sistemas de Salud: principios para una atención integrada. En Informe sobre la salud en el mundo 2003. Forjemos el futuro. Organización Mundial de la Salud; Ginebra. 2003. p. 115-146.

2. Atun R, de Andrade LO, Almeida G, Cotlear D, Dmytraczenko T, Frenz $P$ et al. Health-system reform and universal health coverage in Latin America. Lancet 2015, 385(9974): 1230-1247. http://dx.doi.org/10.1016/S0140-6736(14)61646-9

3. Puig Junoy J, Pinto Prades JL, Ortún Rubio V. El análisis coste-beneficio en sanidad. Aten Primaria 2001; 27(6):422-7. http://dx.doi. org/10.1016/S0212-6567(01)78825-7

4. Starfield B, Shi L, Macinko J. Contribution of Primary Care to Health Systems and Health. Milbank Q. 2005; 83(3):457-502. http://dx.doi. org/10.1111/j.1468-0009.2005.00409.x

5. Macinko J, Starfield B, Shi L. Quantifying the health benefits of primary care physician supply in the United States. Int J Health Serv. 2007;37(1):111-26. http://dx.doi.org/10.2190/3431-G6T7-37M8-P224

6. Macinko J, Starfield B, Shi L. The Contribution of Primary Care Systems to Health Outcomes within Organization for Economic Cooperation and Development (OECD) Countries, 1970-1998. Health Serv Res. 2003; 38(3):831-865. http://dx.doi.org/10.1111/1475-6773.00149

7. Organization for Economic Co-Operation and Development. OECD-STAT[Internet]. Consultado 20 febrero 2018. Disponible en: https:// stats.oecd.org/index.aspx?DataSetCode=HEALTH_STAT\#.

8. The World Bank [Internet]. Consultado 27 febrero 2018.Disponible https://data.worldbank.org/

9. Szwarcwald C L. On the World Health Organization's measurement of health inequalities. J Epidemiol Community Health. 2002;56(3):177182.

10. R Development Core Team (2011), R: A Language and Environment for Statistical Computing. Vienna, Austria: The R Foundation for Statistical Computing. ISBN: 3-900051-07-0. Acceso 18 enero 2018. Disponible en http://www.R-project.org/.

11. Chetty VK; Culpepper L, Phillips RL, Rankin J, Xieral I, Finnegan S et al. FPs Lower Hospital Readmission Rates and Costs. Am Fam Physician. 2011;83(9):1054.

12. Vallejo-Torres L. Morris S. Primary care supply and quality of care in England. Eur J Health Econ. 2018;19(4):499-519. http://dx.doi. org/10.1007/s10198-017-0898-2 
13. Jutz R. The role of income inequality and social policies on income-related health in equalities in Europe. Int J Equity Health. $2015 ; 14: 117$. http://dx.doi.org/10.1186/s12939-015-0247-y

14. Bergqvist K, Yngwe MA, Lundberg O. Understanding the role of welfare state characteristics for health and inequalities - an analytical review. BMC Public Health. 2013; 13:1234. http://dx.doi.org/10.1186/1471-2458-13-1234

15. Navarro V, Shi L. The political context of social inequalities and health. Soc Sci Med. 2001;52(3):481-91. Sociol Health Illn. 2011;33(6):94664.

16. Muntaner C, Borrell C, Ng E, Chung H, Espelt A, Rodriguez-Sanz M, et al. Politics, welfare regimes, and population health: controversies and evidence. Sociol Healt IIIn 2011;33(6): 946-64. http://dx.doi.org/10.1111/j.1467-9566.2011.01339.x

17. Christopher AS, Himmelstein DU, Woolhandler S, McCormick D. The Effects of Household Medical Expenditures on Income Inequality in the United States. Am J Public Health 2018; 108 (3):351-354. http://dx.doi.org/10.2105/AJPH.2017.304213

18. Boing AC, Bertoldi AD, Posenato LG, Peres KG. La influencia de los gastos de salud en el empobrecimiento de los hogares en Brasil. Rev Saude Publica. 2014; 48(5):797-807. http://dx.doi.org/10.1590/S0034-8910.2014048005113

19. HuY, van Lenthe FJ, Mackenbach JP. Income inequality, life expectancy and cause-specific mortality in 43 European countries, 1987-2008: a fixed effects study. Eur J Epidemiol 2015; 30(8):615-625. http://dx.doi.org/10.1007/s10654-015-0066-x

20. Paykani T, Rafiey H, Sajjadi H. A fuzzy set qualitative comparative analysis of 131 countries: which configuration of the structural conditions can explain health better? Int J Equity Health. 2018; 17(1):10. 